\title{
A fatal case of pneumonitis and hypogammaglobulinemia with use of a CDK4/6 inhibitor in metastatic breast cancer: case report
}

\author{
Sara Ashraf ${ }^{1}$, Layana Biglow ${ }^{2}$, Jennifer Dotson $^{1}$, Maria Tria Tirona ${ }^{1}$ \\ ${ }^{1}$ Department of Hematology-Oncology, Joan C Edwards School of Medicine at Marshall University, Huntington, WV, USA; ${ }^{2}$ Department of \\ Internal Medicine, Joan C Edwards School of Medicine at Marshall University, Huntington, WV, USA \\ Correspondence to: Sara Ashraf, MD. Department of Hematology-Oncology, Joan C Edwards School of Medicine at Marshall University, 1400 Hal \\ Greer Blvd, Huntington, WV 25701-3655, USA. Email: saraashraf87@yahoo.com.
}

\begin{abstract}
Breast cancer is the second most common cancer amongst women in the United States following non-melanoma skin cancer. There were an estimated 276,480 new cases and 42,170 deaths in 2020. The lifetime risk for developing breast cancer in females is about $13 \%$. In the United States this year approximately 284,200 people out of which 281,550 women and 2,650 men, will be diagnosed with invasive breast cancer. In recent years, treatment options with novel mechanisms have emerged. Their use in metastatic hormone positive breast cancer has drastically improved outcomes in patients. Cyclin dependent kinase (CDK) 4/6 inhibitors are novel therapies used as adjunct treatments along with hormonal therapy for metastatic breast cancers expressing estrogen receptors (ER) and/or progesterone receptors (PR). These drugs are important regulatory enzymes that target the cell cycle, causing disruption to tumor cell growth. Selective inhibition of CDK4/6 causes cell cycle to arrest in the G1 phase, resulting in reduced cell viability and tumor response. Abemaciclib is the only one approved as monotherapy. Palbociclib and ribociclib must be used along with endocrine therapy such as tamoxifen, aromatase inhibitors or fulvestrant. Common side effects include cytopenias, but rarely, you may see pneumonitis, which is a black box warning. We present a fatal case of severe pneumonitis with superimposed fungal respiratory infection in the setting of hypogammaglobulinemia in a 65-year-old female with metastatic ER and PR positive, HER-2 negative breast cancer who received abemaciclib.
\end{abstract}

Keywords: Pneumonitis; hypogammaglobulinemia; CDK4/6 inhibitor; intravenous immunoglobulin (IVIG); metastatic breast cancer; case report

Received: 15 July 2021; Accepted: 08 November 2021; Published: 31 January 2022.

doi: $10.21037 /$ tbcr-21-19

View this article at: https://dx.doi.org/10.21037/tbcr-21-19

\section{Introduction}

Breast cancer is the second most common cancer amongst women in the United States following non-melanoma skin cancer. There were an estimated 276,480 new cases in 2020, accounting for $15.3 \%$ of all new cancer diagnoses. Breast cancer contributed to 42,170 deaths, which accounts for $7 \%$ of all cancer deaths. The lifetime risk for developing breast cancer in females is about $13 \%$. Six percent of women are stage IV at diagnosis. 5-year survival rate for metastatic breast cancer is $28 \%$. About $30 \%$ of breast cancer survivors will develop metastatic disease at some point (1).
Fortunately, due to extensive ongoing research in the field, there have been many new therapies in the field of metastatic breast cancer which has drastically improved median survival in these patients. Cyclin dependent kinase (CDK) $4 / 6$ inhibitors are a novel therapy in metastatic hormone positive breast cancer, and these include palbociclib, ribociclib and abemaciclib. CDKs are important regulatory enzymes in cell cycle transitions and cell division (2). Tumor suppressor retinoblastoma $(\mathrm{Rb})$ protein controls key transition from growth-1 phase (G1) to the DNA synthesis, or S phase. During G1, a variety of growth signals can result in cyclin-D binding either CDK4 or CDK6 thereby 
causing phosphorylation of $\mathrm{Rb}$ and ultimately the release of E2F (transcription factor genes) and cell cycle progression. Selective inhibition of CDK4/6 causes the cell cycle to arrest in the G1 phase, resulting in reduced cell viability and tumor response (2).

Currently, the mainstay treatment for patients with metastatic hormone positive, HER-2 negative breast carcinoma includes one of the three CDK inhibitors approved by the Food and Drug Administration (FDA), palbociclib, ribociclib, or abemaciclib in combination with endocrine therapy. Hematologic toxicities primarily neutropenia, are common side effects of CDK4/6 inhibitors though usually mild to moderate and managed with dose interruption and/or dose reduction. A rare but serious adverse effect is pneumonitis, a severe inflammation of the lungs. Febrile neutropenia occurs less frequently than neutropenia alone if there is close monitoring of complete blood counts. Pneumonitis is even less common (3). Abemaciclib, the only CDK inhibitor approved for monotherapy, demonstrates greater selectivity for CDK than the other CDK inhibitors. This results in lower rates of hematologic toxicities, and therefore can be dosed continuously, as ribociclib and palbociclib require treatment breaks (3). In this case, we present a patient who developed hypogammaglobulinemia and severe pneumonitis despite dose interruption of abemaciclib. We present the following case in accordance with the CARE reporting checklist (available at https://tbcr.amegroups.com/article/ view/10.21037/tbcr-21-19/rc).

\section{Case presentation}

A 65-year-old female with history of rheumatoid arthritis on hydroxychloroquine and steroids, gastroesophageal reflux disease, hypertension, and metastatic breast cancer presented to the emergency room with fever. She had a history of right-sided ER positive (strong intensity in $98.3 \%$ of cells)/PR positive (strong intensity in $86.2 \%$ of cells), HER-2/neu negative invasive lobular carcinoma diagnosed 5 years prior. She was initially staged as IIB (T3, N0, M0). She had a pre-operative computed tomography (CT) chest with IV contrast that showed subpleural irregular opacities with areas of honeycombing consistent with underlying mild pulmonary fibrosis. She first underwent right partial mastectomy with axillary sentinel node biopsy which revealed an 8.5 -centimeter $(\mathrm{cm})$ tumor with positive margins and 5 out of 6 lymph nodes positive. She was therefore upstaged to IIIA, pT3N2M0. The patient then underwent a right modified radical mastectomy with axillary lymph node dissection. Pathology revealed invasive lobular carcinoma staining estrogen receptor (ER) positive, progesterone receptor (PR) positive, and HER$2 /$ neu negative by immunohistochemistry (IHC). Margins were positive and 16 out of 23 lymph nodes were involved, further upstaging to IIIC (pT3, N3, M0).

After radical mastectomy, she had adjuvant chemotherapy with 21-day cycle for four cycles of dose dense doxorubicin [60 milligram (mg) per meter squared] and cyclophosphamide (600 $\mathrm{mg}$ per meter squared) with pegfilgrastim for marrow convalescence followed by 14-day cycle for four cycles of dose dense paclitaxel (175 mg per meter squared). After completion, she received radiotherapy to the chest wall [5,000 centigray (cGy) with 25 fractions of 100 cGy per day over a course of 48 days], supraclavicular fossa $(5,000 \mathrm{cGy}$ with 25 fractions of $200 \mathrm{cGy}$ per day over a course of 48 days) and a boost to the mastectomy scar (1,000 cGy with 5 fractions of 200 cGy per day over a course of 4 days). Afterwards, the patient was prescribed adjuvant anastrozole $1 \mathrm{mg}$, an aromatase inhibitor, which was taken daily for 5 years along with calcium and vitamin D. She was also started on denosumab as her dual energy $\mathrm{X}$-ray absorptiometry (DEXA) scan revealed osteopenia.

Five years from initial diagnosis, the patient presented to the emergency department (ED) complaining of worsening lower back pain and was found to have numerous osseous metastases on magnetic resonance imaging (MRI) of the lumbar spine. There was no evidence of cord compression. MRI of the cervical and thoracic spine was done which was also positive for metastatic disease. CT guided biopsy of an L2 lesion confirmed metastatic invasive lobular carcinoma of the breast consistent with a breast primary, ER positive, PR negative and HER-2 not overexpressed. Subsequently, she underwent stereotactic body radiation therapy (SBRT) 3,000 cGy in 10 fractions over 14 elapsed days of the L1S2 lesions for palliation. CT chest showed stable post treatment subpleural fibrotic changes in right lung and mild dependent atelectasis and/or subpleural fibrotic change at the left base similar to prior. No inflammatory consolidation, suspicious lung nodule or pleural effusion seen. CT abdomen and pelvis did not reveal any evidence of metastatic disease. After completion of SBRT, the patient was started on fulvestrant (500 $\mathrm{mg}$ intramuscularly) and denosumab (Xgeva $120 \mathrm{mg}$ subcutaneously every 4 weeks) with plans to initiate abemaciclib $150 \mathrm{mg}$ twice a day. Abemaciclib was delayed 2 months due to heightened coronavirus disease 2019 (COVID-19). 
Approximately 1 month after initiating abemaciclib, patient presented to the ED for shortness of breath and fever of 102 Fahrenheit for 5 days. Two weeks prior she was admitted at another hospital where she was treated for pneumonia with intravenous IV vancomycin and ceftriaxone and placement of a bronchial stent. Her symptoms worsened despite completing a course of cefdinir. At the time of admission, the complete blood count revealed white blood cell count (WBC) 5.0 thousand/cubic millimeter $\left(\mathrm{k} / \mathrm{mm}^{3}\right)$ [normal range (NR), $4.5-10.0 \mathrm{k} / \mathrm{mm}^{3}$ ], hemoglobin (Hgb) 10.1 grams/deciliter (g/dL) (NR, 12.0-16.0 g/dL), platelet count $105 \mathrm{~g} / \mathrm{dL}$ (NR, 150-440 g/dL), absolute neutrophil count (ANC) $2.3 \mathrm{k} / \mathrm{mm}^{3}$ (NR, $2.0-8.0 \mathrm{k} / \mathrm{mm}^{3}$ ), absolute lymphocyte count (ALC) $1.9 \mathrm{k} / \mathrm{mm}^{3}$ (NR, $1.0-4.8 \mathrm{k} / \mathrm{mm}^{3}$ ), blood urea nitrogen (BUN) $11.0 \mathrm{mg} / \mathrm{dL}$ (NR, 7.0-18.0 mg/dL) and creatinine $1.22 \mathrm{mg} / \mathrm{dL}(\mathrm{NR}$, $0.60-1.10 \mathrm{mg} / \mathrm{dL})$. A chest X-ray revealed patchy upper lobe and peripheral predominant airspace disease. She was started on IV vancomycin and piperacillin/tazobactam. Subsequent CT of the chest revealed significant infiltration in both upper lobes having the appearance of pneumonia. Emphysematous changes and interstitial fibrotic changes seen in both lower lobes with an inflammatory or edematous superimposed process. There was no evidence of metastatic disease or lymphangitic spread.

After further questioning, the patient had recently discontinued her abemaciclib due to ongoing worsening symptoms after 2 weeks, including hypoxic respiratory failure requiring supplemental oxygen via nasal cannula.

The patient was started on corticosteroids and bronchodilators. Blood cultures were negative at 5 days, viral respiratory PCR was negative for viral pathogens and sputum cultures, all obtained on admission, were contaminated with oropharyngeal flora. At that time, ANC was $1.28 \mathrm{k} / \mathrm{mm}^{3}$. Serum immunoglobulin levels were measured to further investigate the etiology of patient's worsening respiratory status. Total immunoglobulin G (IgG) measured 163 milligram/deciliter $(\mathrm{mg} / \mathrm{dL})$ (NR, 553-1,360 mg/dL) IgA level was $53 \mathrm{mg} / \mathrm{dL}$ (NR, $71-374 \mathrm{mg} / \mathrm{dL}$ ), and all subtypes of IgG were decreased. There were no baseline immunoglobulin levels to compare since there was never an indication to check them. Human immunodeficiency virus (HIV) was negative. Patient was treated with one dose of $90 \mathrm{~g}$ of intravenous immunoglobulin (IVIG) and was also started empirically on trimethoprim-sulfamethoxazole due to concern for possible Pneumocystis jirovecii pneumonia (PJP). Both hypogammaglobulinemia and severe lymphopenia were attributed to possible immunosuppression due to chronic steroid use, or a sequela of CDK4/6 inhibitor causing severe hypogammaglobulinemia. The patient's respiratory status worsened requiring five liters of oxygen via nasal cannula and a chest X-ray showed low lung volumes and bilateral chronic appearing opacities. Bronchoscopy with bronchoalveolar lavage and cultures was recommended by pulmonary, but patient declined to undergo the procedure. Subsequently, the patient's hospital course was complicated by atrial fibrillation with rapid ventricular response and decline in her respiratory status, requiring high flow oxygen via nasal cannula, followed by bilevel positive airway pressure (BIPAP). Additional pertinent laboratory testing during that time revealed Fungitell level $250 \mathrm{pg} / \mathrm{mL}$, ANC $19.5 \mathrm{k} / \mathrm{mm}^{3}$ and ALC $0.6 \mathrm{k} / \mathrm{mm}^{3}$. The patient decided to pursue comfort measures and passed away shortly thereafter. All procedures performed in this study were in accordance with the ethical standards of the institutional and/or national research committee(s) and with the Helsinki Declaration (as revised in 2013).

Written informed consent was waived as the patient was a widow who passed away more than a year ago. The patient's consent was not obtained as the author cannot reach her family by the numbers listed on patient's chart.

\section{Discussion}

We report a fatal case of severe lung injury with presumed superimposed fungal respiratory infection in a 65 -yearold female with metastatic ER positive/HER2 negative breast cancer who received abemaciclib $150 \mathrm{mg}$ twice a day for 10 days. The patient did have a history of asymptomatic underlying mild pulmonary fibrosis due to rheumatoid arthritis (Figures 1,2), but after administration of the CDK4/6 inhibitor, the patient's respiratory status deteriorated significantly, and lung imaging revealed extensive interstitial pulmonary scarring (Figure 3,4). In this case, we surmise that there is correlation between the usage of CDK4/6 inhibitors and subsequent clinical complications which led to the patient's demise. The use of a CDK4/6 inhibitor likely led to the resultant neutropenia, lymphopenia and cellular immunodeficiency which in turn brought on a severe infection. The patient also had evidence for pneumonitis, or inflammatory reaction in the lungs, which is an uncommon side effect related to this class of drugs.

There is a plethora of data linking anti-neoplastic medications to pulmonary toxicity. Commonly used anti- 


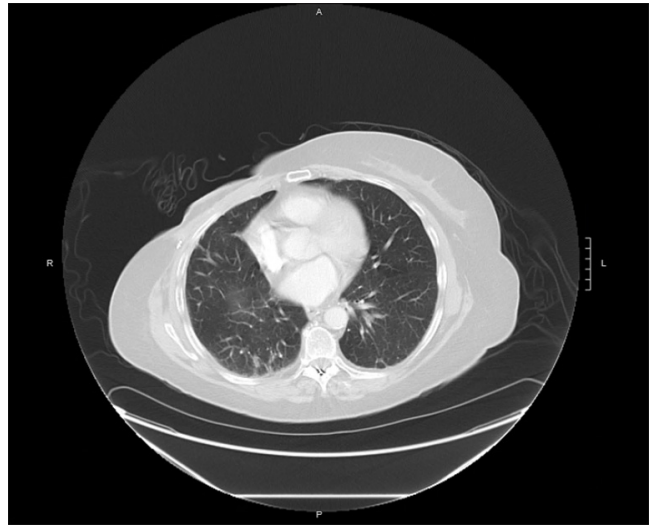

Figure 1 Computerized tomography (CT) chest performed before starting abemaciclib; shows mild pulmonary fibrosis in a nonspecific pattern with calcified hilar and mediastinal lymph nodes.

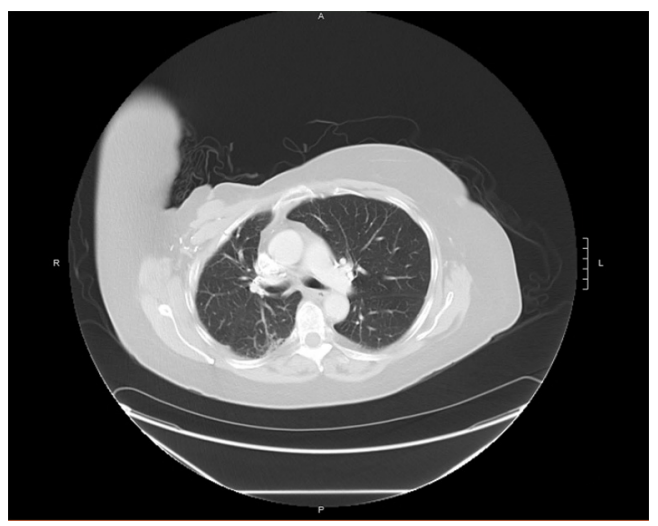

Figure 2 Computerized tomography (CT) chest performed before starting abemaciclib; shows mild pulmonary fibrosis in a nonspecific pattern with calcified hilar and mediastinal lymph nodes.

cancer drugs associated with drug induced lung injury include gemcitabine, docetaxel, bleomycin and immune check point inhibitors. There is a scarcity of data regarding prevalence of pneumonitis or severe lung injury linked to CDK4/6 inhibitors, likely due to their relatively recent usage. In the MONARCH 3 trial with abemaciclib in metastatic breast cancer, there was one death reported which was attributed to pneumonitis (4). In a case report describing the use of abemaciclib in metastatic breast cancer, Ahsan et al. reported development of respiratory

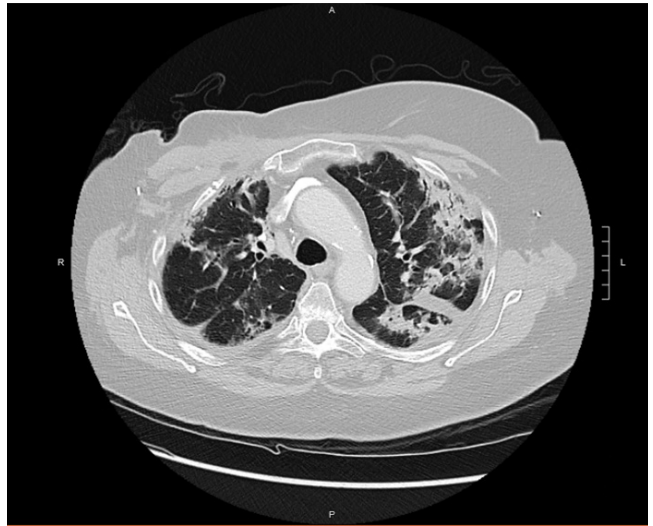

Figure 3 Computed tomography (CT) performed approximately 3 months post abemaciclib therapy; shows significant pulmonary infiltration in bilateral upper lobes and emphysematous changes with interstitial fibrotic changes more pronounced compared to previous studies.

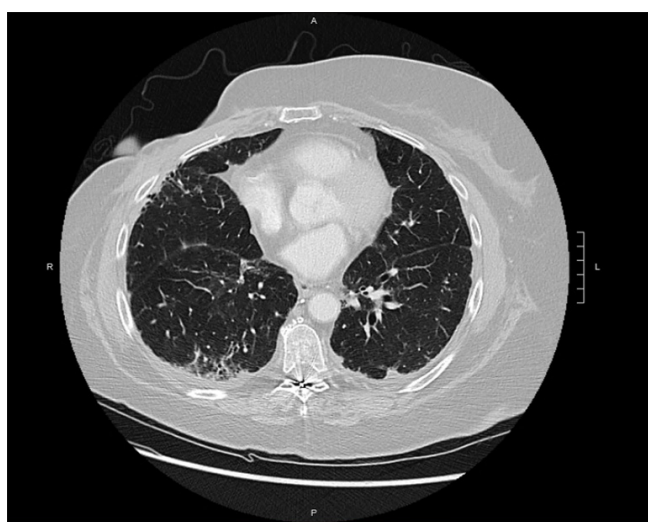

Figure 4 Computed tomography (CT) performed approximately 3 months post abemaciclib therapy; shows significant pulmonary infiltration in bilateral upper lobes and emphysematous changes with interstitial fibrotic changes more pronounced compared to previous studies.

distress and ground glass opacities in a 52-year-old woman with non-revealing infectious work-up and no prior pulmonary disease, which was ultimately fatal (5). Another case presented by Gong et al. reported development of pneumonitis after eight cycles of palbociclib in combination with fulvestrant which resolved with conservative management and discontinuation of the CDK4/6 inhibitor (6). Our patient had evidence of mild pulmonary fibrosis at baseline, which may have predisposed her to develop chemical pneumonitis. Presumably, our patient 
likely developed pneumonia secondary to neutropenia, lymphopenia and hypogammaglobulinemia which may have been multi-factorial related to CDK4/6 inhibitor as well as chronic immunosuppression due to treatment of her underlying rheumatoid arthritis.

Throughout the literature, the most common hematologic side effect of CDK4/6 inhibitors was neutropenia, which can be explained by the integral role of CDK6 in proliferation of hematologic precursors. CDK4/6 inhibitors lead to the cytostatic effect on neutrophil precursors resulting in pharmacologic quiescence. Most cases of CDK4/6 inhibitor induced neutropenia are mild and have an uncomplicated course. It is usually managed with dose interruption and/or dose reduction to prevent future neutropenic episodes (3). Throughout our patient's hospitalization, the ANC fluctuated, with the lowest at $1.028 \mathrm{k} / \mathrm{mm}^{3}$ but at the time of patient's death, ANC was elevated at $19.59 \mathrm{k} / \mathrm{mm}^{3}$, which did not correlate with likely opportunistic superinfection. Beta-D-glucan was elevated at $250 \mathrm{pg} / \mathrm{mL}$, which may suggest underlying fungal infections such as candidiasis and aspergillosis, but the specificity of this assay is limited with a high false positive rate (7).

With deterioration in her clinical status, further investigation revealed hypogammaglobulinemia, in addition to her known neutropenia and lymphopenia. CDK4/6 are cofactors for cyclin D3 which plays a role in expansion of immature thymocytes/lymphocytes. Their inhibitors therefore lead to severe lymphopenia. Circulatory IgG and IgM antibodies are usually found in response to fungal antigens in individuals with symptomatic systemic fungal infections such as aspergillosis, blastomycosis, candidiasis, cryptococcosis and histoplasmosis (8). The patient's inability to mount an effective immune response to a presumably latent fungal infection is due to several causes including the essential immunodeficiency characterized by hypogammaglobulinemia and lymphopenia.

Hypogammaglobulinemia has mostly been associated with B-cell depleting antineoplastic therapy such as rituximab in the literature. Most of the studies in the review defined hypogammaglobulinemia as a serum IgG less than 5.65 to $7 \mathrm{~g}$ per deciliter. A major risk factor found in the literature review included low baseline low levels of IgG and $\mathrm{IgM}$, with persistent hypogammaglobulinemia defined as hypogammaglobulinemia lasting more than 6 months after treatment with rituximab; these patients also developed the more severe bronchopulmonary infections (9). There is little to no data showing any association of hypogammaglobulinemia associated with CDK inhibitors, while neutropenia and severe interstitial pneumonitis were reported in initial clinical trials.

Neutropenia occurred in $41 \%$ of patients receiving abemaciclib plus an aromatase inhibitor in MONARCH 3, $46 \%$ of patients receiving abemaciclib plus fulvestrant in MONARCH 2, and 37\% of patients receiving single agent abemaciclib in MONARCH 1. Febrile neutropenia was reported in less than $1 \%$ of patients receiving abemaciclib in all MONARCH studies $(3,4,10)$.

Severe, life threatening or fatal interstitial lung disease (ILD) and/or pneumonitis can occur in patients receiving abemaciclib and other CDK4/6 inhibitors. In MONARCH 1, MONARCH 2 and MONARCH 3, 3.3\% of patients developed ILD of any grade, $0.6 \%$ had grade 3 or 4 , and $0.4 \%$ were fatal. Dose interruption or reduction is recommended for patients who develop recurrent or persistent grade 2 ILD and permanent discontinuation for grade 3 or $4(3,4,10)$.

In MONARCH 2, 43\% of patients receiving abemaciclib and fulvestrant developed infections of all grades, $5 \%$ had grade 3 and less than $1 \%$ grade 4 . Placebo plus fulvestrant arm had $25 \%$ of all grades, $3 \%$ grade 3 and less than $1 \%$ grade 4. Lymphopenia of all grades was found in $63 \%$ of patients given abemaciclib and fulvestrant, $12 \%$ had grade 3 and less than $1 \%$ grade 4 . Whereas placebo and fulvestrant arm had $32 \%$ lymphopenia of all grades, $2 \%$ grade 3 and $0 \%$ grade 4 (9).

\section{Conclusions}

Although CDK inhibitor induced lung injury is documented as a rare side effect in PALOMA and MONARCH 3, there is very little data about how these medications affect those with underlying pulmonary fibrosis. One should strongly consider screening patients with underlying rheumatologic disorder for baseline pulmonary scarring before starting any CDK4/6 inhibitors, as the drug-induced pneumonitis may be life threatening, as seen in our patient, who rapidly deteriorated. It was also observed in clinical trials that palbociclib can induce severe lymphopenia and severe immunodeficiency characterized by decreased lymphocyte count, more specifically decreased CD4 T lymphocytes. It should be acknowledged that abemaciclib can not only have similar effects, but can also cause life threatening hypogammaglobulinemia, leading to opportunistic fungal infections and worsening of pneumonitis and vice versa. Consider monitoring lymphocyte count and immunoglobulin levels in patients receiving abemaciclib. 
There may be a role for antifungal prophylaxis in conjunction with PJP prophylaxis. Physicians should be cautious about the risk of lymphopenia especially in patients with autoimmune disorders. In patients who develop hypogammaglobulinemia, those with an autoimmune disorder have higher infection rates than those without. Thorough post marketing surveillance needs to be done to evaluate this further and raise awareness about the potential relationship between hypogammaglobulinemia, pneumonitis and CDK4/6 inhibitors.

\section{Acknowledgments}

Funding: None.

\section{Footnote}

Reporting Checklist: The authors have completed the CARE reporting checklist. Available at https://tbcr.amegroups. com/article/view/10.21037/tbcr-21-19/rc

Conflicts of Interest: All authors have completed the ICMJE uniform disclosure form (available at https://tbcr. amegroups.com/article/view/10.21037/tbcr-21-19/coif). The authors have no conflicts of interest to declare.

Ethical Statement: The authors are accountable for all aspects of the work in ensuring that questions related to the accuracy or integrity of any part of the work are appropriately investigated and resolved. All procedures performed in studies were in accordance with the ethical standards of the institutional and/or national research committee(s) and with the Helsinki Declaration (as revised in 2013). Written informed consent was waived as the patient was a widow who passed away more than a year ago. The patient's consent was not obtained as the author cannot reach her family by the numbers listed on patient's chart.

Open Access Statement: This is an Open Access article distributed in accordance with the Creative Commons Attribution-NonCommercial-NoDerivs 4.0 International License (CC BY-NC-ND 4.0), which permits the noncommercial replication and distribution of the article with the strict proviso that no changes or edits are made and the original work is properly cited (including links to both the formal publication through the relevant DOI and the license).
See: https://creativecommons.org/licenses/by-nc-nd/4.0/.

\section{References}

1. SEER Cancer Stat Facts: Female Breast Cancer. National Cancer Institute. Bethesda, MD. Available online: https:// seer.cancer.gov/statfacts/html/breast.html

2. Spring LM, Wander SA, Zangardi M, et al. CDK 4/6 Inhibitors in Breast Cancer: Current Controversies and Future Directions. Curr Oncol Rep 2019;21:25.

3. Dickler MN, Tolaney SM, Rugo HS, et al. MONARCH 1, A Phase II Study of Abemaciclib, a CDK4 and CDK6 Inhibitor, as a Single Agent, in Patients with Refractory HR+/HER2- Metastatic Breast Cancer. Clin Cancer Res 2017;23:5218-24.

4. Goetz MP, Toi M, Campone M, et al. MONARCH 3: Abemaciclib As Initial Therapy for Advanced Breast Cancer. J Clin Oncol 2017;35:3638-46.

5. Ahsan I, Malik F, Jafri S. Palbociclib related pneumotoxicity: a rare side effect. Am J Respir Crit Care Med 2017;195:A5546

6. Gong J, Cho M, Yu KW, et al. A single institution experience with palbociclib toxicity requiring dose modifications. Breast Cancer Res Treat 2018;168:381-7.

7. Ostrosky-Zeichner L, Alexander BD, Kett DH, et al. Multicenter clinical evaluation of the (1-->3) beta-Dglucan assay as an aid to diagnosis of fungal infections in humans. Clin Infect Dis 2005;41:654-9.

8. Lanternier F, Cypowyj S, Picard C, et al. Primary immunodeficiencies underlying fungal infections. Curr Opin Pediatr 2013;25:736-47.

9. Sacco KA, Abraham RS. Consequences of B-cell-depleting therapy: hypogammaglobulinemia and impaired B-cell reconstitution. Immunotherapy 2018;10:713-28.

10. Sledge GW Jr, Toi M, Neven P, et al. MONARCH 2: Abemaciclib in Combination With Fulvestrant in Women With HR+/HER2- Advanced Breast Cancer Who Had Progressed While Receiving Endocrine Therapy. J Clin Oncol 2017;35:2875-84.

doi: $10.21037 /$ tbcr-21-19

Cite this article as: Ashraf S, Biglow L, Dotson J, Tirona MT. A fatal case of pneumonitis and hypogammaglobulinemia with use of a CDK4/6 inhibitor in metastatic breast cancer: case report. Transl Breast Cancer Res 2022;3:9. 